\section{Prevalencia del carcinoma epidermoide en el Servicio de Cirugía Cabeza y Cuello del Hospital Nacional Dos de Mayo durante el periodo 2005-2016}

\author{
Prevalence of squamous cell carcinoma in \\ the Head and Neck Surgery Service of the \\ National Hospital Dos de Mayo during the \\ period 2005-2016
}

\begin{abstract}
Resumen
Objetivo: Evaluar la prevalencia del carcinoma epidermoide en la cavidad oral en el Servicio de Cirugía de Cabeza y Cuello de un hospital peruano durante el periodo 20052016. Métodos: Estudio descriptivo, retrospectivo y transversal. Se incluyeron 4269 biopsias desde enero del 2005 hasta diciembre del 2016, obtenidas de las historias clínicas del Servicio de Cirugía de Cabeza y Cuello del Hospital Dos de Mayo. El diagnóstico de carcinoma epidermoide se obtuvo de la base de datos del Servicio de Anatomía Patológica. Se determinó la prevalencia del carcinoma epidermoide con los datos obtenidos dividiendo el total de individuos con dicha enfermedad entre el total de la población biopsiada durante ese periodo. Resultados: Se obtuvo un total de 4269 biopsias durante el periodo 2005-2016, de las cuales 80 biopsias presentaron diagnóstico anatomopatológico de carcinoma epidermoide en la cavidad oral. La prevalencia del carcinoma epidermoide en la cavidad oral fue de 1,87\%, afectando más al sexo femenino, con una relación aproximada de 1,4:1; respecto al sexo masculino. Excepto entre los años 2009 al 2012 en los que hubo una mayor predilección por el sexo masculino. El mayor número de casos (28 pacientes) se presentó en pacientes de 41 a 50 ańos, seguido de 31 a 40 años (16 pacientes) y 61 a 70 ańos (14 pacientes). En cuanto a la localización, el sitio más predispuesto a la presencia de esta lesión cancerígena fue la región de la lengua, seguido del paladar y el reborde alveolar/encía. Conclusiones: La prevalencia del carcinoma epidermoide fue de $1,87 \%$. Afectó más al sexo femenino, en la quinta década de vida y se localizó con mayor frecuencia en la región de la lengua.
\end{abstract}

Palabras clave: Carcinoma epidermoide; Cavidad oral; Prevalencia.

\begin{abstract}
Objective: To evaluate the prevalence of squamous cell carcinoma in the oral cavity in the Head and Neck Surgery Service of a Peruvian hospital during the period 2005-2016. Methods: Descriptive, retrospective, cross-sectional study. We included 4269 biopsies from January 2005 to December 2016, adquired from the records of the Head and Neck Surgery Service of the Dos de Mayo Hospital, Lima, Peru. The diagnosis of squamous cell carcinoma was obtained from the database of the pathological anatomy service of said hospital. The prevalence of squamous cell carcinoma was determined by dividing the total of individuals with said disease over the total biopsied population during that period. Results: From a total of 4,269 biopsies obtained during the period 2005-2016;
\end{abstract}

\section{Artículo Original}

Angel Frank Delesma Chumbe ${ }^{1, a}$

1 Universidad Nacional Mayor de San Marcos,

Facultad de Odontología. Lima, Perú.

${ }^{a}$ Cirujano Dentista

\section{Correspondencia:}

Angel Frank Delesma Chumbe

Correo electrónico: delesma@hotmail.com

Santa Cruz Mz E Lt 14 Los Olivos, Lima, Perú.

Conflicto de intereses: Los autores declaran no tener conflictos de interés.

Fuente de financiamiento: Autofinanciado

Fecha de recepción: 15/02/18

Fecha de aceptación: 10/05/18 
80 biopsies were identified with anatomopathological diagnosis of squamous cell carcinoma in the oral cavity. The prevalence of squamous cell carcinoma in the oral cavity was $1.87 \%$. More female than male patients, were affected; in an approximate ratio of 1.4 : 1, except between 2009 and 2012, when there was a greater prevalence on male patients. The largest number of cases (28 patients) with squamous cell carcinoma in the oral cavity occurred in patients 41-50 years old, followed by 31-40 years old (16 patients), 61-70 years old (14 patients). Regarding the location of squamous cell carcinoma in the oral cavity, it was manifested mostly in the region of the tongue, followed by the palate, alveolar ridge/ gingiva. Conclusions: The prevalence of squamous cell carcinoma was $1.87 \%$. Females were more affected. It manifested itself more in the 5th decade of life. The location of squamous cell carcinoma in the oral cavity was mostly in the region of the tongue.

Keywords: Epidermoid carcinoma; Mouth; Prevalence.

\section{Introducción}

El cáncer oral es uno de los tumores más comunes en todo el mundo y representa aproximadamente el 3\% de todas las neoplasias malignas en ambos sexos ${ }^{1,2}$.

La Organización Mundial de la Salud estima mayor prevalencia de cáncer oral en lugares como: Asia sudoriental, Europa, Mediterráneo oriental, América, África y la región del Pacifico Occidental ${ }^{3}$.

El carcinoma de células escamosas o carcinoma epidermoide es la neoplasia maligna más común y representa el $90 \%$ de todos los cánceres de la cavidad oral. En la lengua se presenta aproximadamente del $25 \%$ al $40 \%$, produciendo disfunciones en el habla, masticación y deglución ${ }^{4-6}$. Afecta principalmente a las personas adultas entre 55 a 75 ańos. Su etiopatogenia es desconocida, pero implica una serie de factores de riesgo como los genéticos, químicos, infecciosos, físicos, enfermedades preexistentes, deficiencias nutricionales, etc. La incidencia a nivel mundial está directamente relacionada con los hábitos de consumir tabaco y alcohol ${ }^{7,8}$.

Las formas precoces pueden presentarse clínicamente como placas leucoplásicas o eritroleucoplásicas, puede tener un pequeño crecimiento tumoral, o más frecuentemente como una ulceración que cura fácilmente. En su periodo inicial es local y curable, pero conforme avanza el tiempo se disemina a tejidos adyacentes sanos, invade los ganglios linfáticos del cuello disminuyendo enormemente la posibilidad de curación. Todas las tumoraciones o ulceraciones de la cavidad oral que no desaparezcan en dos semanas deben ser biopsiadas y enviadas para un diagnóstico histológico. La biopsia excisional, indicada para tumores de dimensión reducida; o incisional, indicada cuando se extirpa un fragmento de la periferia de la misma junto con tejido sano ${ }^{9-11}$.

El tratamiento quirúrgico, según criterio de la escuela americana, depende del grado de malignidad del tumor, la ubicación y del tamaño en diferentes partes de la lengua en que el tumor se puede localizar. Esta escuela menciona que tumores pequeños y de regular tamaño sin metástasis ganglionares se favorecen con la extirpación amplia y completa, junto con la radioterapia post operatoria en caso en que los bordes de sección resulten infiltrados por la neoplasia. Con respecto a los carcino- mas de gran tamaño, especialmente los que comprometen la base de la lengua, se obtienen mejores resultados con el tratamiento de radioterapia y quimioterapia ${ }^{12,13}$.

Por todo lo expuesto, el propósito de la presente investigación fue determinar la prevalencia del carcinoma epidermoide en la cavidad oral en el Servicio se Cirugía Cabeza y Cuello de un hospital durante el periodo 2005-2016.

\section{Métodos}

Se diseñó un estudio descriptivo, retrospectivo y transversal que evaluó la prevalencia del carcinoma epidermoide en la cavidad oral. La muestra de estudio estuvo conformada por todos los pacientes de los cuales se obtuvieron 4269 biopsias del Servicio de Cirugía Cabeza y Cuello del Hospital Nacional Dos de Mayo durante el periodo 2005-2016, Lima - Perú.

Los datos se recolectaron en una ficha y las variables analizadas fueron: sexo, edad, zona de ubicación de la biopsia, año y diagnóstico anatomopatológico de carcinoma epidermoide, el cual fue emitido por el Servicio de Anatomía Patológica de dicho hospital. Una vez que se recolectó la información se elaboró una base de datos. Los resultados fueron analizados utilizando el paquete estadístico SPSS versión 21.

\section{Resultados}

Se registraron un total de 4269 biopsias, de los cuales 80 obtuvieron el diagnóstico histopatológico de carcinoma epidermoide durante el periodo 2005 al 2016 emitidas por el Departamento de Anatomía Patológica (Tabla 1 y Figura 1). La prevalencia del carcinoma epidermoide fue de $1,87 \%$.

Según el sexo, el carcinoma epidermoide afectó en mayor medida a las mujeres, observándose una relación aproximada de 1,4:1 con respecto al sexo masculino, excepto entre los años 2009 al 2012 en los que hubo una mayor predilección por el sexo masculino (Figura 2).

Según la edad, el mayor número de casos con carcinoma epidermoide en la cavidad oral se presentó en pacientes que están en la quinta década de vida (28 pacientes), seguido de la cuarta, séptima, octava, sexta, novena y tercera década de vida (Figura 3). 
Tabla 1. Prevalencia de carcinoma epidermoide en la cavidad oral por año, en pacientes atendidos en el Servicio de Cirugía de Cabeza y Cuello del Hospital Nacional Dos de Mayo en el periodo 2005-2016

\begin{tabular}{|c|c|c|c|c|c|c|c|c|c|c|c|c|c|}
\hline Año & 2005 & 2006 & 2007 & 2008 & 2009 & 2010 & 2011 & 2012 & 2013 & 2014 & 2015 & 2016 & Total \\
\hline $\mathrm{N}^{\circ}$ Biopsias & 384 & 380 & 370 & 377 & 330 & 219 & 342 & 235 & 353 & 371 & 398 & 510 & 4269 \\
\hline Frecuencia & 10 & 6 & 11 & 11 & 5 & 6 & 4 & 6 & 7 & 1 & 11 & 2 & 80 \\
\hline $\begin{array}{l}\text { Prevalencia carcino- } \\
\text { ma epidermoide (\%) }\end{array}$ & 12,5 & 7,5 & 13,75 & 13,75 & 6,25 & 7,5 & 5 & 7,5 & 8,751 & 1,25 & 13,75 & 2,5 & 100 \\
\hline
\end{tabular}

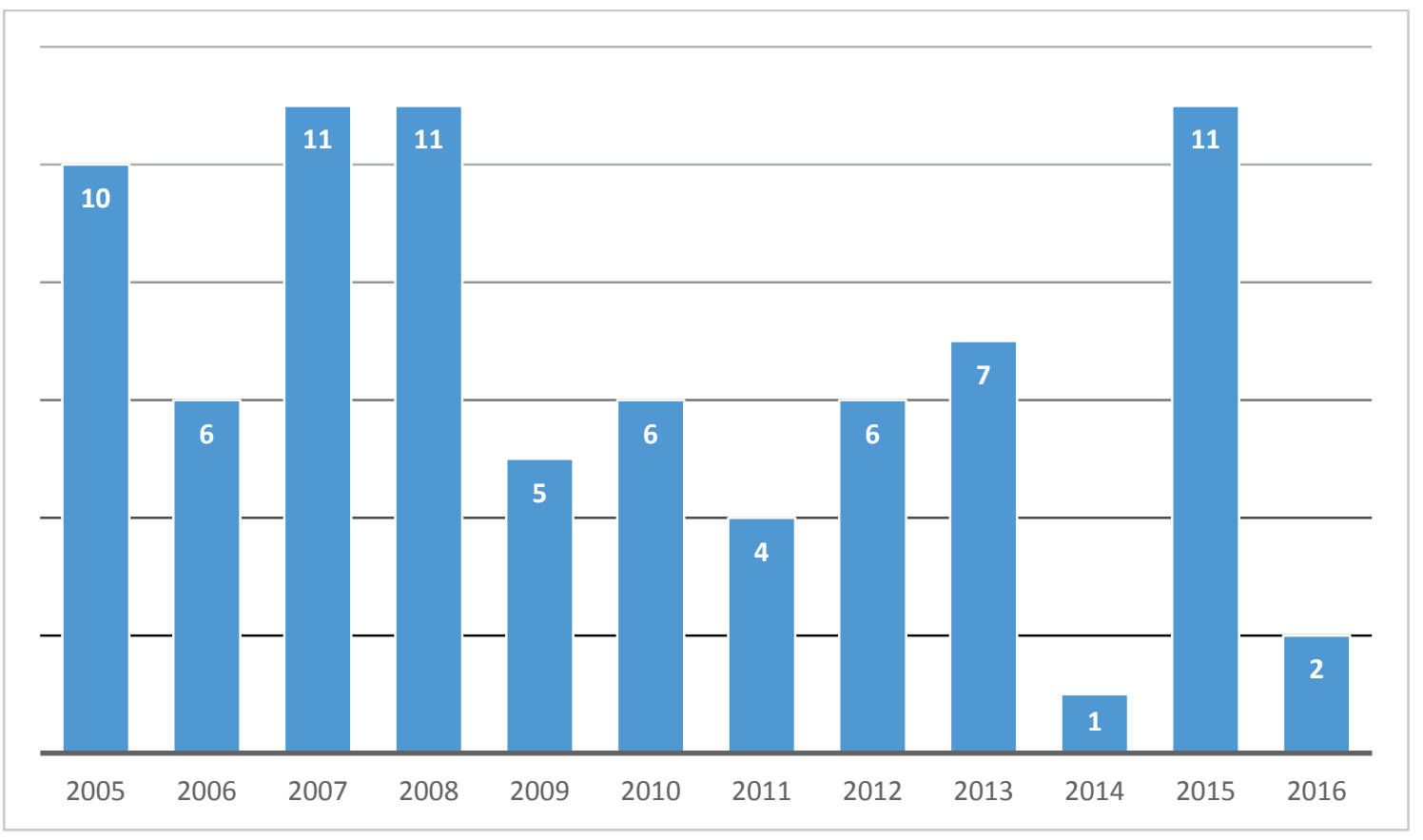

Figura 1. Distribución de carcinoma epidermoide en la cavidad oral en pacientes atendidos en el Servicio de Cirugía de Cabeza y Cuello del Hospital Nacional Dos de Mayo, 2005-2016

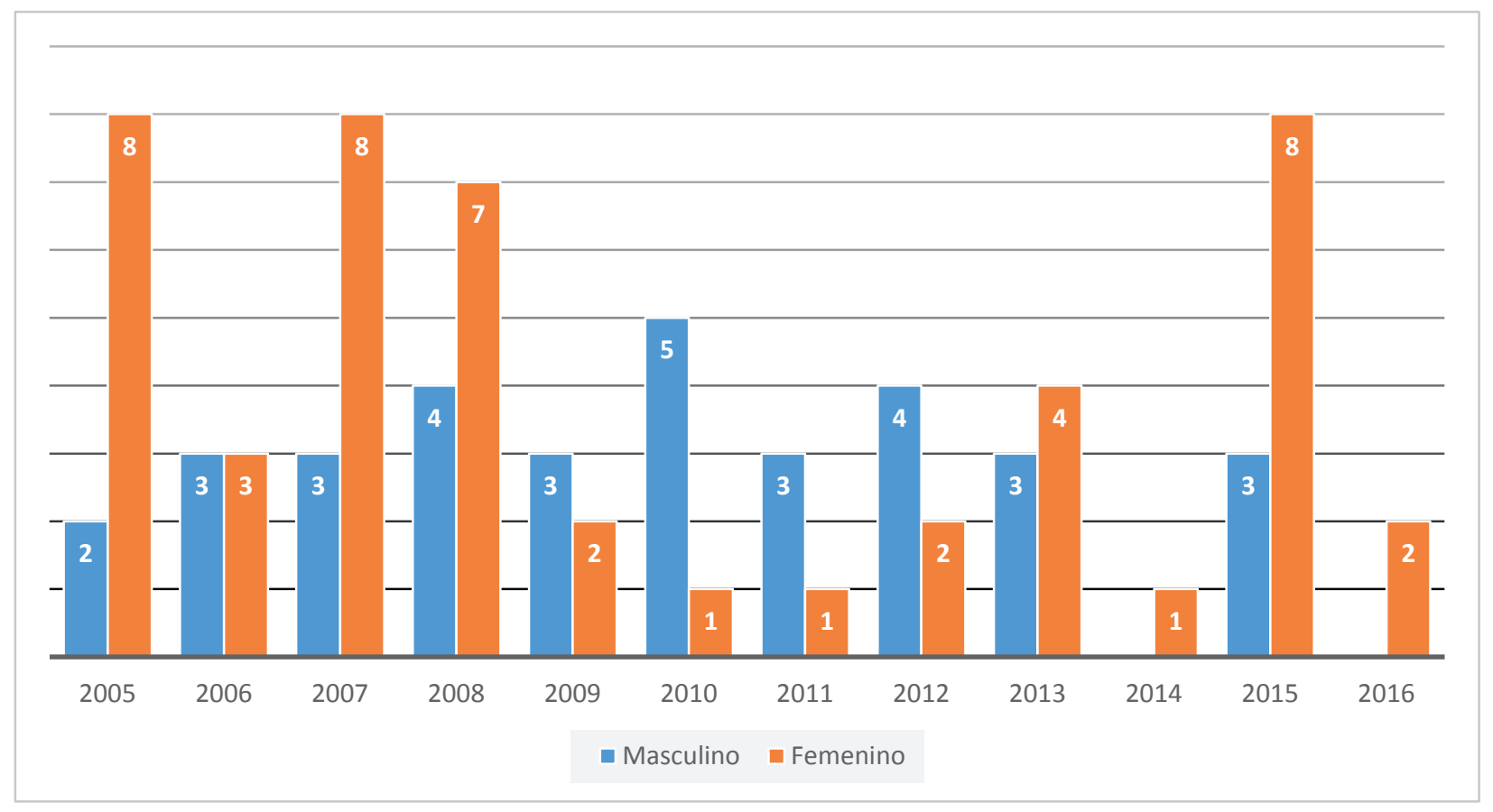

Figura 2. Distribución del carcinoma epidermoide en la cavidad oral por sexo, en pacientes atendidos en el Servicio de Cirugía de Cabeza y Cuello del Hospital Nacional Dos de Mayo, 2005 al 2016 


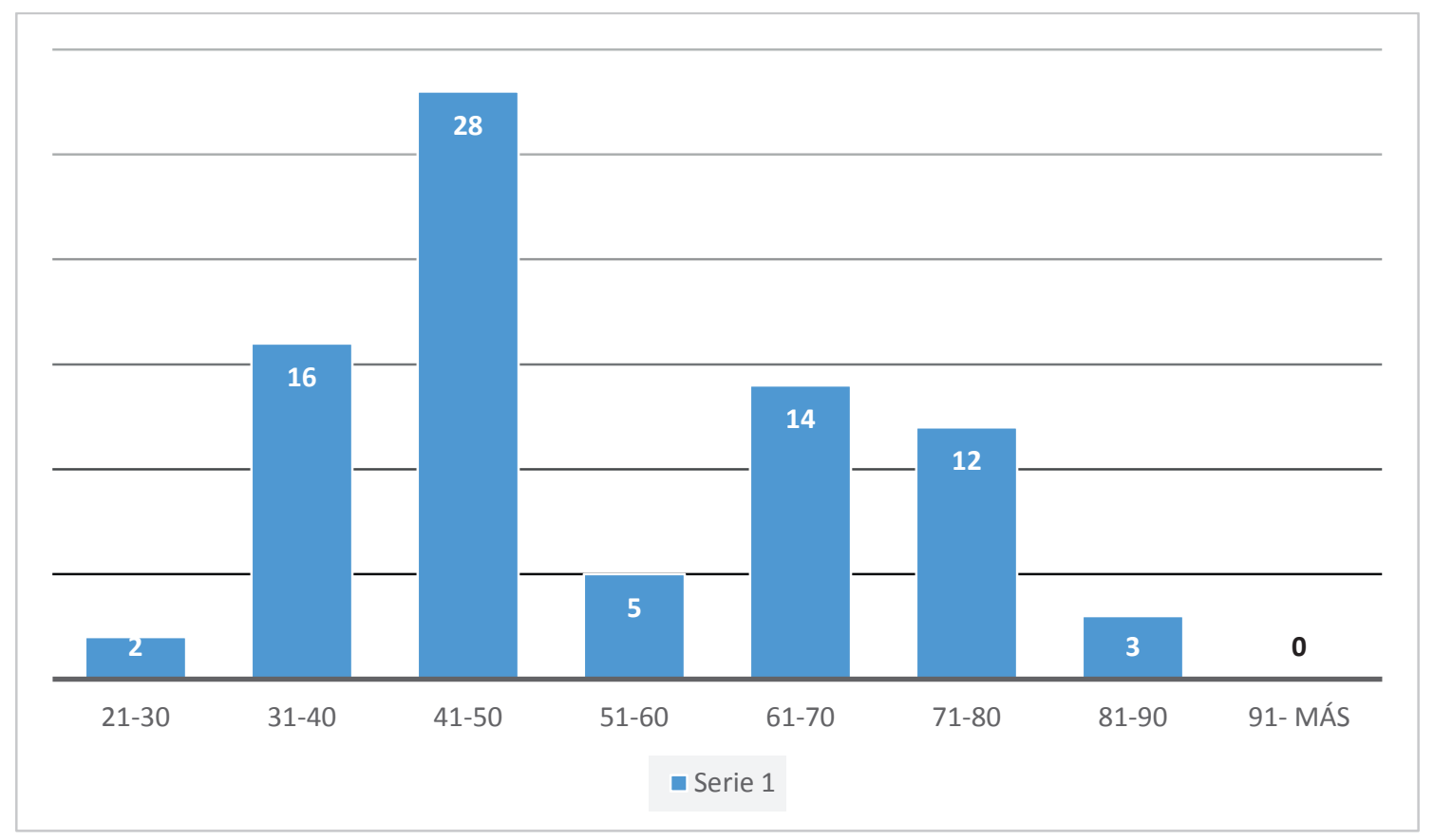

Figura 3. Distribución del carcinoma epidermoide en la cavidad oral según la edad de los pacientes atendidos en el Servicio de Cirugía de Cabeza y Cuello del Hospital Nacional Dos de Mayo, 2005-2016

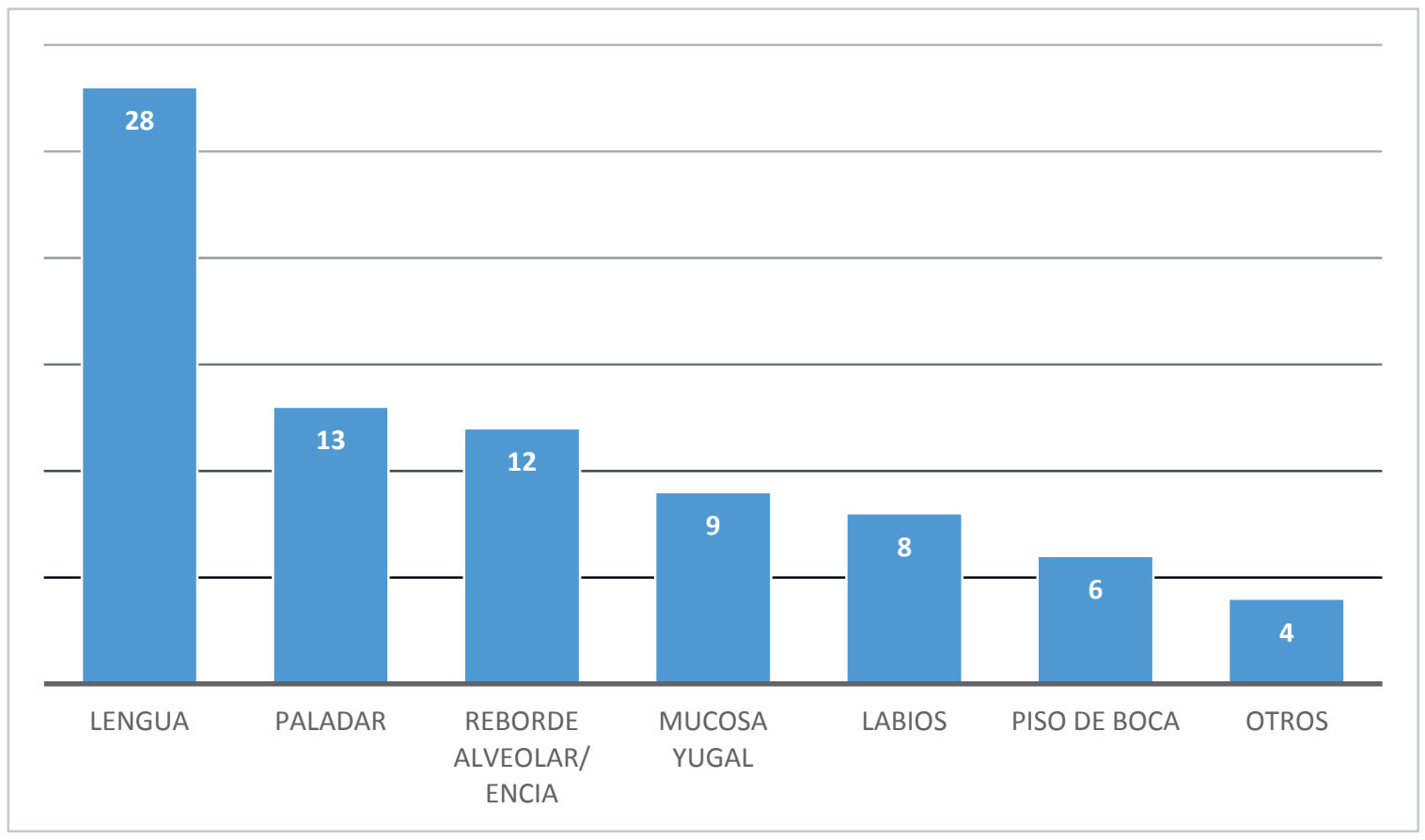

Figura 4. Distribución del carcinoma epidermoide en la cavidad oral según la ubicación anatómica en los pacientes atendidos en el Servicio de Cirugía de Cabeza y Cuello del Hospital Nacional Dos de Mayo, 2005-2016

La localización del carcinoma epidermoide en la cavidad oral se manifestó en su mayoría en la región de la lengua, seguido del paladar, reborde alveolar/encía, mucosa yugal, labios y piso de boca (Figura 4).

\section{Discusión}

Según el trabajo realizado, el sexo femenino fue el más afectado en una relación de 1,4:1 coincidiendo con los trabajos realizados por García ${ }^{14}$, Urresti ${ }^{15}$ y García ${ }^{16}$; quienes encontraron una relación entre mujeres y hombres de $(1,2: 1),(1,6: 1)$ y $(1: 1)$; respectivamente. Sin em- bargo, Frías et al. ${ }^{17}$ y Ceccotti et al. ${ }^{18}$ indicaron que el sexo masculino es el más afectado. Esto se puede deber a los factores de riesgo como tabaco y alcohol, cuyo consumo ha aumentado en los últimos tiempos en el sexo femenino ${ }^{19,20}$.

Según la edad, García ${ }^{14}$, Urresti ${ }^{15}$ y García ${ }^{16}$ reportaron una mayor frecuencia de carcinoma epidermoide en la séptima década de vida. Frias et al. ${ }^{17}$ encontraron que el $50 \%$ de los casos correspondían a personas mayores de 60 ańos. Según la presente investigación, la quinta década de vida fue la más afectada; esto puede deberse 
a los cambios que sufre la mucosa oral con el paso del tiempo además de la longevidad, debido a que pierde la capacidad para reparar los daños producidos en los tejidos, esto hace que aumente su vulnerabilidad frente a la acción de los agentes carcinógenos ${ }^{21,22}$.

Estudios realizados por Urresti ${ }^{15}$, García ${ }^{16}$, Frías et al. ${ }^{17}$, Ceccotti et al. ${ }^{18}$ y Coronado ${ }^{23}$ indicaron una mayor afección del carcinoma epidermoide en la lengua. Estas investigaciones coinciden con nuestros resultados, esto puede deberse a que la mucosa de la lengua está tapizado por una fina capa de epitelio escamoso en contraste al paladar duro y reborde alveolar que poseen un epitelio mucho más grueso, esto le brinda una mayor protección contra el trauma mecánico y la acción de productos carcinógenos que al disolverse en la saliva tienden a aglomerarse en zonas gravitatorias como el piso de la boca y la zona ventrolateral de la lengua ${ }^{24-26}$.

Se concluye que la prevalencia del carcinoma epidermoide fue de $1,87 \%$, el sexo femenino fue el más afectado y se manifestó más en la quinta década de vida. La localización del carcinoma epidermoide en la cavidad oral se evidenció con mayor frecuencia en la región de la lengua. El aumento de los factores de riesgo en los últimos tiempos, fundamentalmente el consumo tabaco y alcohol, especialmente por el sexo femenino pueden producir alteraciones a nivel oral que perjudican el estilo de vida. A medida que se realicen más estudios de carcinoma epidermoide en el país se obtendrá una mejor realidad del problema.

\section{Agradecimientos}

El autor agradece al Hospital Nacional Dos de Mayo y a los jefes de los diferentes Servicios de dicho hospital: Dr. Franscisco Avello Canisto - Jefe del Servicio de Cabeza y Cuello. Dr. Alejandro Alfaro Lozano - Jefe del Servicio Anatomía Patológica.

\section{Referencias bibliográficas}

1. Chi AC, Day TA, Neville BW. Oral cavity and oropharyngeal squamous cell carcinoma an update. CA Cancer J Clin. 2015;65(5):401-21. DOI: 10.3322/caac.21293.

2. Scully C, Bagan J. Oral squamous cell carcinoma overview. Oral Oncol.2009;45(4):301-8. DOI: 10.1016/j. oraloncology.2009.01.004

3. Ferlay J, Soerjomataram I, Dikshit R, Eser S, Mathers C, Rebelo M, et al. Cancer incidence and mortality worldwide: sources, methods and major patterns in GLOBOCAN 2012. Int J Cancer. 2015;136(5):359-86. DOI: 10.1002/ijc.29210.

4. Kimple AJ, Welch CM, Zevallos JP, Patel SN. Oral cavity squamous cell carcinoma-an overview. Oral Health Dent Manag. 2014;13(3):877-82.

5. García GV, Gonzáles MA, Bascones MA. Bases moleculares del cáncer oral. Revisión bibliográfica. Av. Odontoestomatología. 2005;21(6):287-95.

6. Yu ZW, Zhong LP, Ji T, Zhang P, Chen WT, Zhang CP. MicroRNAs contribute to the chemoresistance of cisplatin in tongue squamous cell carcinoma lines. Oral
Oncol. 2010;46(4):317-22. DOI: 10.1016/j.oraloncology.2010.02.002.

7. Caribé GF, Chimenos E, López J, Finestres F, Guix B. Manejo odontológico de las complicaciones de la radioterapia y quimioterapia en el cáncer oral. Med Oral. 2003;8(3):178-87.

8. Okazaki Y, Tanaka Y, Tonogi M, Yamane G. Investigation of environmental factors for diagnosing malignant potencial in oral epithelial displasia. Oral Oncology. 2002;38(2):562-73. DOI: 10.1016/S09156992(07)80022-7

9. Tonaco BF, Saliba GC, Carvalhosa AA, Souza PH, Costa LR. Epidemiologia do câncer de boca em laboratorio público do Estado de Mato Grosso, Brasil. Cad. Saúde Pública, Rio de Janeiro. 2008;24(9):1977-82.

10. Rivera SP, Martínez RB. Morbilidad y mortalidad por cáncer oral y faríngeo en Chile. Rev Méd Chile. 2005;133(5):555-63.

11. Cosio DH, Lazo OL. Biopsia en Odontología. Rev SITUA. 2006;15(2):37-41.

12. Ow TJ, Myers JN. Current management of advanced resectable oral cavity squamous cell carcinoma. Clin Exp Otorhinolaryngol. 2011;4(1):1-10. DOI: 10.3342/ ceo.2011.4.1.1

13. Spiro RH, Strong EW. Discontinuos partial glosectomy and radical neck dissection in selected patients with epidermoide carcinoma of the mobile tongue. Am J Surg.1973;126(4):544-546. DOI: 10.1016/S00029610(73)80047-9

14. García EZ. Prevalencia de cáncer en mucosa oral en el servicio de estomatología quirúrgica del hospital nacional Arzobispo Loayza en el período 2008 al 2012.[Tesis de Bachiller]. Lima (Perú): Facultad de Odontología, Universidad Nacional Mayor de San Marcos; 2014.

15. Urresti JE. "Frecuencia y distribución del cáncer oral en sujetos adultos mayores evaluados en el instituto de enfermedades neoplásicas "Dr. Eduardo Cáceres Graziani" (1990 - 1999)". [Tesis de Bachiller]. Lima (Perú): Facultad de Estomatología, Universidad Peruana Cayetano Heredia, 2003.

16. García AO. Prevalencia de carcinoma epidermoide oral en pacientes de dos hospitales de salud pública-Perú en el periodo 2006-2009. [Tesis de Bachiller]. Lima (Perú): Facultad de Odontología, Universidad Nacional Mayor de San Marcos, 2010.

17. Frías MM, Zeichner GI, Súchil BL, Ochoa CF. Epidemiologia descriptiva de cáncer de cavidad bucal en el lnstituto Nacional de Cancerología (1985-1992). Rev Inst Nal Cancerol. 1997;43(2):80-85.

18. Ceccotti EL, Sforza RR, Carzoglio JC, Luberti R, Flichman JC. El Diagnóstico en Clínica Estomatológica. 1 ra edición.Ed. Médica Panamericana; 2007.

19. Johnson NW, Bain CA. Tobacco Intervention: Tobacco and oral disease. Br Dent J. 2000;189(4):200-206. DOI: 10.1038/sj.bdj.4800721

20. Teng A, Atkinson J, Disney G, Wilson N. Changing smoking-mortality association over time and across social groups: National census-mortality cohort studies 
from 1981 to 2011. Scip Rep.2017;7(1):1-10. DOI: 10.1038/s41598-017-11785-x.

21. García JM, Lence AJ, Sosa RM. Mortalidad del cáncer bucal en Cuba (1987-1996). Rev Cuba Oncol. 1999;15(2):114-18.

22. Almeida RM. Incidencia del carcinoma epidermoide en cavidad oral. Lima. [Tesis de Bachiller]. Lima (Perú): Facultad de odontología, Universidad Nacional Federico Villareal,1995.

23. Coronado TR. Prevalencia de Cáncer de lengua y su manejo quirúrgico en el Hospital Nacional Arzobispo
Loayza. [Tesis de Bachiller]. Lima (Perú): Facultad de odontología, Universidad Nacional Mayor de San Marcos, 2004.

24. Robbins S, Coltran, R. Patología Estructural y Funcional. 7ma edición. Madrid: ELSEVIER; 2009.

25. Neville B, Damm D, Allen C, Bouquot J. Oral and Maxillofacial Pathology. 3rd ed. Philadelphia: Elseviers Saunders; 2009.

26. Regezi J, Sciubba J, Jordan R. Oral Pathology: Clinical Pathologic Correlaions.7th ed.USA: Elsevier Health Sciences; 2015. 